\title{
Expression and significance of Parkinson disease protein 7 in placental, serum and umbilical cord blood in preeclampsia
}

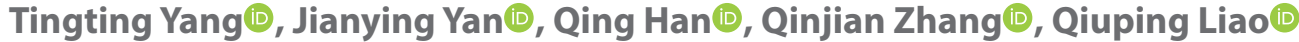 \\ Department of Obstetrics and Gynecology, Fujian Maternity and Child Health Hospital, Affiliated Hospital of Fujian \\ Medical University, Fujian, China
}

\begin{abstract}
Objectives: To study the role of changes in the expression of human Parkinson's disease protein 7 (PARK7/DJ-1) in preeclampsia.

Material and methods: We selected 120 gravidas, including 60 cases of severe preeclampsia group and control group, and divided into early onset preeclampsia group ( $<34$ weeks), late onset preeclampsia group ( $\geq 34$ weeks) and control group according to the onset of pregnancy. The expression level of DJ-1 was detected by ELISA. The expression level of DJ-1 in placenta tissue of gravidas was detected by Western-blot and RT-PCR.

Results: The level of DJ-1 in serum and cord blood of preeclampsia group was higher than that of control group. The relative level of DJ-1 protein and DJ-1 mRNA in placenta tissue of preeclampsia group was higher than that of control group.

Conclusions: The expression level of DJ-1 in serum, umbilical cord blood and placenta tissue increased in preeclampsia patients, suggesting that DJ-1 may take part in the pathophysiology process of preeclampsia.
\end{abstract}

Key words: DJ-1; preeclampsia; placenta; cord blood; oxidative stress

Ginekologia Polska 2020; 91, 12: 764-768

\section{INTRODUCTION}

Preeclampsia (PE) is a specific disease in pregnancy. It is the main cause of the incidence and death of gravidas and neonates [1]. The global morbidity is about 3\% to $5 \%$ [2], and about $18 \%$ of pregnancy related deaths are caused by preeclampsia [3]. However the pathophysiological mechanisms of PE remain poorly known. Zhou et al. [4] showed that excessive oxidative stress leads to uterine spiral artery remodeling and superficial placental implantation, which can lead to the onset of preeclampsia. DJ-1, also known as PARK 7, is a multifunctional protein implicated in familial Parkinsonism, Oxidative stress is the main function of DJ-1. Kwon et al. [5] found DJ-1 in placenta of patients with preeclampsia is widespread and high expression, and mainly localized in trophoblast cells, which is closely related with the pathogenesis of preeclampsia, that may be involved in the pathogenesis of DJ-1 in preeclampsia. Thus, the aim of this study was to determine the difference of DJ-1 expression in serum, umbilical cord blood and placenta of women with normal and preeclamptic pregnancies.
Preeclampsia (PE) is a specific disease in pregnancy. It is the main cause of the incidence and death of gravidas and neonates. The global morbidity is about 3\% to $5 \%$, and about $18 \%$ of pregnancy related deaths are caused by preeclampsia. However, the changes of preeclampsia are complex and uncertain. The etiology and pathogenesis are not clear. In recent years, the study found that excessive oxidative stress causes uterine spiral arteries remolding and placental shallow implantation, which can lead to the onset of preeclampsia. Parkinson disease protein 7 (PARK7/DJ-1) is a member of the ThiJ/Pfpl/DJ-1 superfamily, antioxidant stress is the main function of DJ-1. Kwon HS, etc., found that DJ-1 is widely distributed and highly expressed in placental tissues of preeclampsia, and is mainly located in the chorionic trophoblast cells closely related to preeclampsia, and that DJ-1 may participate in pathogenic process of preeclampsia. In this study, we detected the changes in expression of DJ-1 in gravidas' serum, umbilical cord blood and placenta tissues, and investigation the role of it in the pathogenesis of preeclampsia. 


\begin{tabular}{l|l|l|l|l|}
\hline \multicolumn{5}{|l}{ Table 1. Subject clinical characteristics (mean \pm SD) } \\
\hline & Early-onset preeclampsia & Late-onset preeclampsia & Early control group & Late control group \\
\hline Age [years] & $32.7 \pm 5.3$ & $30.2 \pm 5.2$ & $29.7 \pm 4.2$ & $30.9 \pm 3.6$ \\
\hline Gestational age [weeks] & $32.6 \pm 2.9$ & $38.0 \pm 1.8$ & $31.4 \pm 3.0$ & $38.9 \pm 1.1$ \\
\hline SBP $[\mathrm{mm} \mathrm{Hg}]$ & $165.4 \pm 15.4$ & $162.3 \pm 16.8$ & $115.0 \pm 9.5$ & $117.5 \pm 9.8$ \\
\hline DBP $[\mathrm{mm} \mathrm{Hg}]$ & $104.5 \pm 10.7$ & $100.3 \pm 9.4$ & $70.2 \pm 6.7$ & $74.1 \pm 6.9$ \\
\hline MAP $[\mathrm{mm} \mathrm{Hg}]$ & $124.8 \pm 11.1$ & $121.0 \pm 10.9$ & $85.1 \pm 6.2$ & $88.6 \pm 6.8$ \\
\hline Pre-pregnancy BMI [kg/m²] & $22.4 \pm 4.1$ & $22.4 \pm 4.1$ & $20.6 \pm 3.1$ & $22.0 \pm 2.3$ \\
\hline Pregnancy BMI $\left[\mathrm{kg} / \mathrm{m}^{2}\right]$ & $28.3 \pm 4.3$ & $28.8 \pm 3.8$ & $26.4 \pm 2.8$ & $28.0 \pm 2.7$ \\
\hline
\end{tabular}

BMI — body mass index; DBP — diastolic blood pressure; SBP — systolic blood pressure; MAP — mean arterial pressure

\section{MATERIAL AND METHODS}

\section{Patients}

Sixty women with severe preeclampsia and 60 normal pregnant women were enrolled. They were admitted to Fujian Provincial Maternity and Children Hospital, affiliated hospital of Fujian Medical University from November 2015 to March 2017 for delivery. They were divided into four groups: early-onset group, late-onset group, early control group and late control group. PE was defined as hypertension (systolic blood pressure $\geq 140 \mathrm{mmHg}$ and diastolic blood pressure $\geq 90 \mathrm{~mm} \mathrm{Hg}$ after 20 weeks' gestation) and proteinuria ( $\geq 300 \mathrm{mg}$ in a $24 \mathrm{~h}$ urine collection or one dipstick measurement of $\geq 1+$ ) [6]. The clinical situations are summarized in Table 1. Informed consent was signed by each participant, and the study was approved by the ethics committee of Fujian Medical University.

\section{Specimen collection and enzyme-linked immunosorbent assay}

The specimen collocation was similar to Yan et al. [7]. Blood samples were collected from the peripheral veins of all the women. Umbilical cord blood was collected from the umbilical vein immediately after delivery. Placental tissue was collected from the root of the umbilical cord immediately after cesarean section. The DJ-1 ELISA Kit was purchased from XiTang (Shanghai, China). The measurement was performed following the manual.

\section{Western blot}

Protein from the placental tissue was transferred to PVDF membranes and incubated with monoclonal rabbit anti-GAP$\mathrm{DH}$ (1:1500; Abcam, UK) antibodies overnight at $4^{\circ} \mathrm{C}$, then followed by incubation with HRP-conjugated secondary antibodies. After extensive washing, proteins of interest were detected by enhanced chemiluminescence system (ECL, Thermo Scientific, UK) and quantified by densitometry using Quantity One.

\section{RT-PCR}

Total RNA was extracted from placental tissue with a TRIzol reagent kit (Invitrogen, CA, USA). DJ-1 mRNAs and the internal standard (glyceraldehydes 3-phosphate dehydrogenase (GAPDH)) expressions were quantified by real-time polymerase chain reaction (PCR). Primer sequence was as follows, sense 5'-GTAGCCGTGATGTGGTCATTT-3', antisense 5'-CTGTGCGCCCAGATTACCT-3'. PCR assays were performed following the manual. The results were normalized to GAPDH expression levels.

\section{Statistical analysis}

Statistical analysis was performed using one-way ANOVA with Levene test and unpaired t-test using SPSS18.0. Pearson correlation analysis was used to analyze the correlations. $\mathrm{P}<0.05$ was considered as significant.

\section{RESULTS}

\section{Maternal serum DJ-1}

As shown in Figure 1A, maternal serum DJ-1 was $1.6040 \pm 0.465 \mathrm{ng} / \mathrm{L}$ in the early-onset preeclampsia group and $1.2810 \pm 0.419 \mathrm{ng} / \mathrm{L}$ in the late-onset preeclampsia group. Preeclampsia DJ-1 was significantly higher compared to $1.0698 \pm 0.333 \mathrm{ng} / \mathrm{L}$ in the early control group and $1.0625 \pm 0.329 \mathrm{ng} / \mathrm{L}$ in the late control group $(\mathrm{p}<0.05)$. between the early-onset preeclampsia group and the late-onset preeclampsia group was significantly different.

\section{Umbilical cord blood DJ-1}

As shown in Figure 1B, the DJ-1 umbilical cord blood level in the early-onset preeclampsia group is significantly different compared to the late-onset preeclampsia group. Both the early-onset preeclampsia group $(1.3928 \pm 1.036 \mathrm{ng} / \mathrm{L})$ and the late-onset preeclampsia group $(1.4544 \pm 0.695 \mathrm{ng} / \mathrm{L})$ had significantly higher mean DJ-1 compared with the late control group $(0.9764 \pm 0.390 \mathrm{ng} / \mathrm{L})$.

\section{Protein Expression of DJ-1 in placental tissue}

As shown in Figure 2, the DJ-1 expression in placenta was significantly higher in the early-onset preeclampsia group $(1.15 \pm 0.26)$, and late-onset preeclampsia group $(1.50 \pm 0.45)$, compared to late control $(0.82 \pm 0.47 ; p<0.05)$. 
A

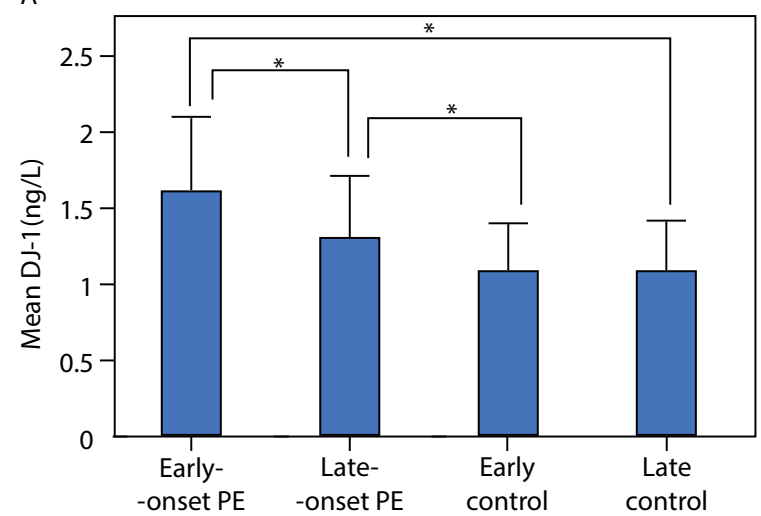

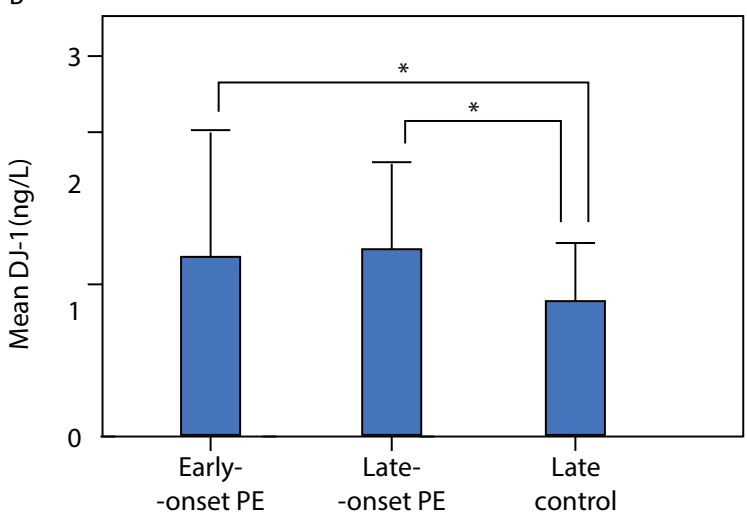

Figure 1. Mean DJ-1 in (A) maternal serum and (B) umbilical cord blood. PE, preeclampsia
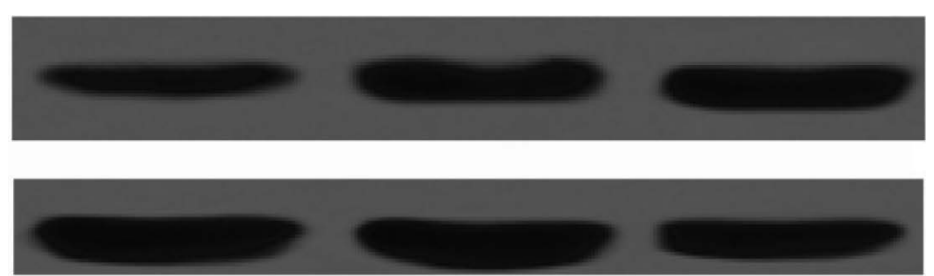

C

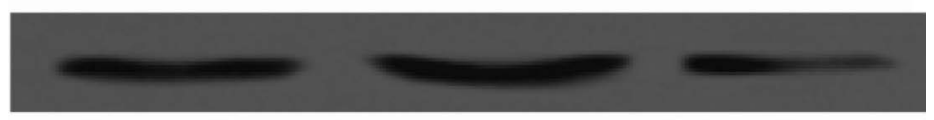

D

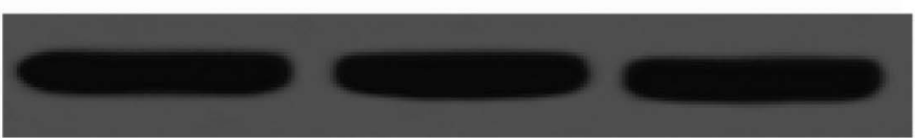

Figure 2. Expression of DJ-1 in placenta tissue for (A) early-onset preeclampsia, (B) late-onset preeclampsia, (C) the late control group and (D) $\beta$-actin

\section{MRNA expression of DJ-1 in placental tissue}

As shown in Figure 3, the mRNA expression of DJ-1 in placenta was significantly higher in the early-onset preeclampsia group (3.315 \pm 1.08$)$, and late-onset preeclampsia group (3.608 \pm 1.87$)$, compared to late control (1.5463 \pm 0.409$)$.

\section{Correlation analysis}

The umbilical cord blood DJ-1 in the early-onset preeclampsia group and late-onset preeclampsia group correlated negatively with Neonatal birth weight $(r=-0.448$ and $r=-0.648, p<0.05)$. There is no significant correlation between the control groups (Fig. 4)

\section{DISCUSSION \\ Preeclampsia and oxidative stress}

Oxidative stress reflects an imbalance between the overproduction and incorporation of free radicals and the dynamic ability of a biosystem to detoxify reactive intermediates [8]. Once this balance is broken, the body's ROS production increases, excessive accumulation of ROS will cause oxidation reactions, resulting in destruction of cell

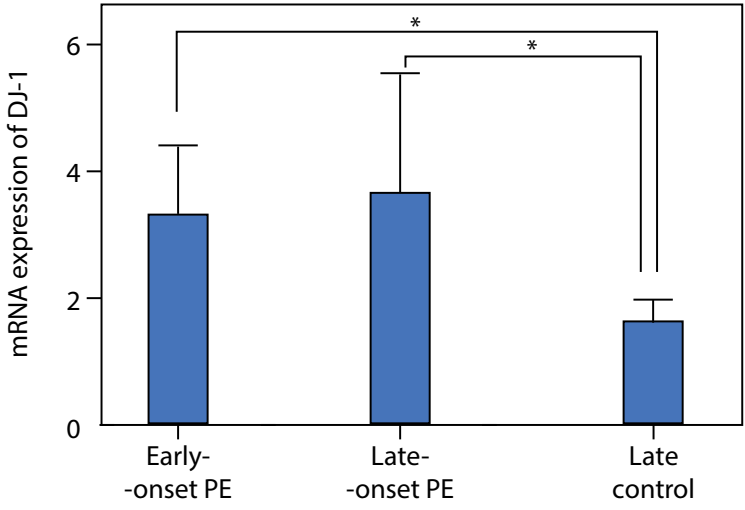

Figure 3. DJ-1 mRNA expression in human placentas from normal and preeclamptic pregnancies. In reverse transcriptase-polymerase chain reaction (RT-PCR), mRNA of DJ-1 was significantly increased in preeclamptic placenta $(p<0.05)$

structure and function, and participate in the occurrence of disease [9]. The oxidation and antioxidant system exists in a relatively balanced state within the normal pregnancy mother [10]. Numerous studies have described elevated 
A

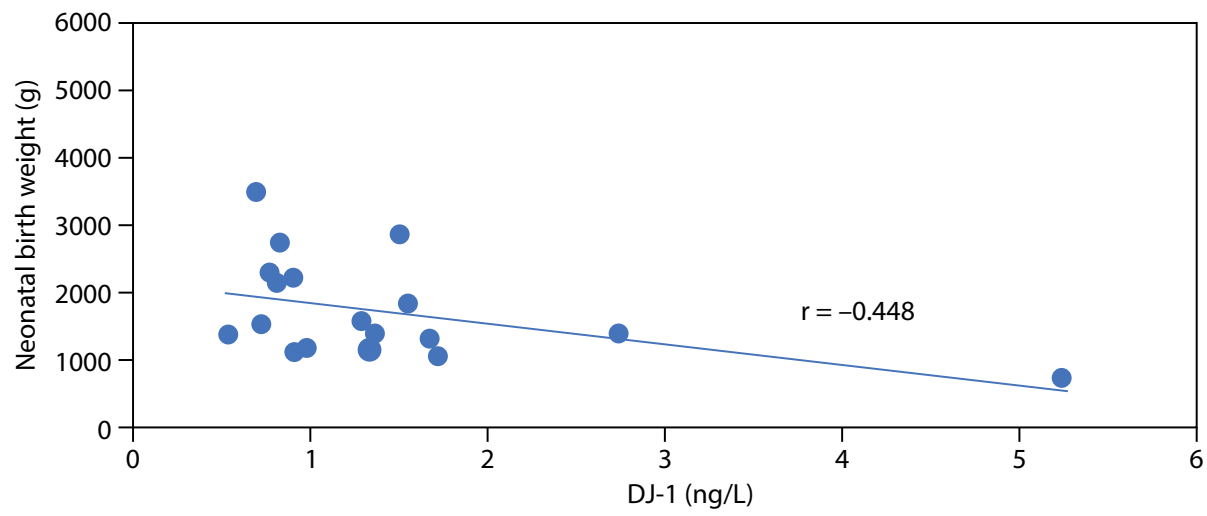

B

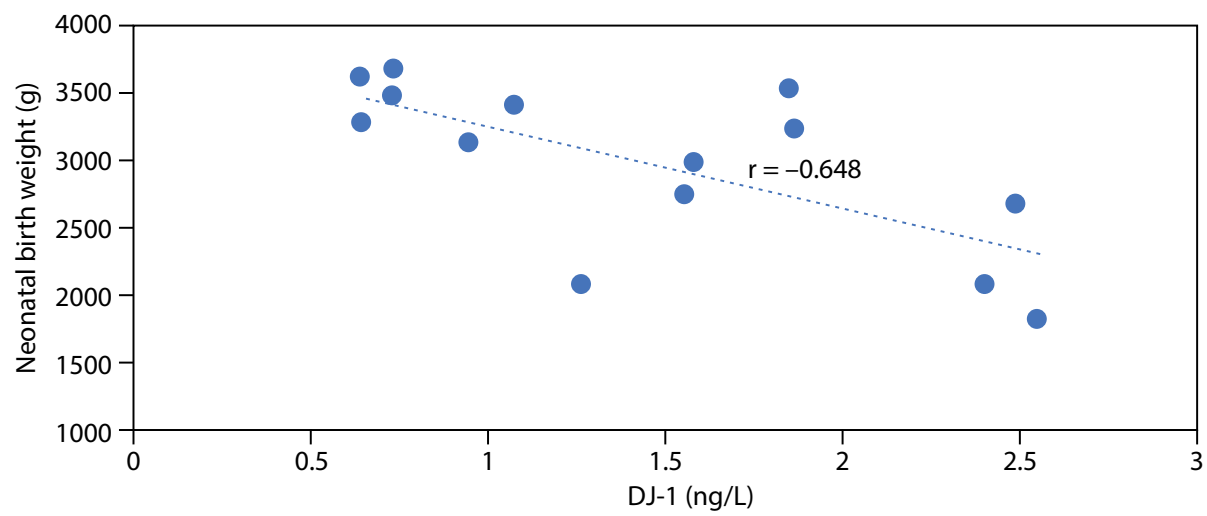

Figure 4. Umbilical cord blood DJ-1 vs Neonatal birth weight in (A) early onset and (B) late-onset preeclampsia

markers of oxidative stress in placental tissue from women with preeclampsia [11]. Ding Det al. [12] confirmed placental trophoblast cells mitochondria increased ROS production in preeclampsia, indicating that excessive ROS injury to the mitochondrial DNA of placenta trophoblast cells so as to involve in the pathogenesis of diseases. The possible role of late in pregnancy, the placenta of patients with preeclampsia decreased blood perfusion, maternal compensatory mechanisms lead to reperfusion injury induced by maternal placenta, produce excess ROS oxidative stress caused by injury [13]. Excessive oxidative stress may lead to extravillous trophoblast (EVT) inhibited proliferation, invasion and apoptosis decreased, the uterine spiral artery recasting failure and shallow placental implantation, promote the pathogenesis of preeclampsia [14].

\section{DJ-1 and preeclampsia}

DJ-1 was first proposed as a mitochondrial dependent oncogene, and recent studies have demonstrated that $D J-1$ is a multifunctional protein that is ubiquitously expressed in more than 20 tissues of the human body. Antioxidant stress is the main function of DJ-1. Normally DJ-1 is highly conserved during biological evolution. In oxidative stress, DJ-1 can oxidize itself to protect cells against ROS induced apoptosis, and the isoelectric point of DJ-1 is reduced from 6.2 to 5.8 [15], DJ-1 is thought to be involved in oxidative stress. The main mechanisms of DJ-1 oxidative stress are: 1) DJ-1 enhances the antioxidant capacity by stabilizing and inhibiting nuclear factor erythroid 2-related factor(Nrf2)proteins Keap1 and the subsequent ubiquitination of Nrf2; 2) DJ-1 can change the oxidative stress related enzyme activities involved in oxidative stress [16]; 3) DJ-1 in order to remove ROS, which is oxidative stress products, will be oxidized by itself, thus losing normal resistance to apoptosis. Although the pathogenesis of preeclampsia has not yet been determined, with the deepening of research for many years, scholars at home and abroad have put forward some reasonable and scientific theories about its etiology and pathogenesis, among which oxidative stress is an important theory.

Kwon HS et al Study on the expression of DJ-1 in preeclampsia placenta tissue and localization. The results showed that preeclampsia trophoblast cells on the positive staining of DJ-1 protein increased significantly compared with the control group, and the high expression of DJ-1 in trophoblast cells. The research also confirmed in placental tissue of preeclampsia on DJ-1 mRNA than the normal group. The incidence of DJ-1 increased the expression of thought in preeclampsia, mainly on placental trophoblastic cells, 
caused by the oxidative stress injury induced by placental dysfunction [6]. The relative expression level of DJ-1 based on Western-blot and RT-PCR was detected in the placenta, the levels were increased compared with the control group the expression of DJ-1 in the placenta of preeclampsia group, and the difference is statistically significant. And the results are consistent. But the specific mechanism of DJ-1 in preeclampsia remains to be explored.

\section{Correlation between umbilical cord blood DJ-1 expression and neonatal birth weight}

The intrauterine growth and development of fetus can be judged by the birth weight of newborns. To some extent, low birth weight reflects the severity of fetal growth restriction. The results of the study showed that preeclampsia group neonatal body mass was significantly better than the control group were decreased significantly, the correlation analysis found that umbilical cord blood DJ-1 expression level and neonatal birth weight was negatively correlated, the expression level of DJ-1 in umbilical cord blood and fetal growth and development are closely related. A possible mechanism for the patients with preeclampsia due to uterine spiral artery remodeling abnormal trophoblast shallow invasion to ischemia, hypoxia, anti-angiogenesis and angiogenic factors in the body to lose balance. This imbalance is not conducive to angiogenesis leads to widespread endothelial dysfunction, the trophoblast cells around in a relatively hypoxic state, thus the generation of oxidative stress, the effects of nutrient transport to the fetus, fetal growth restriction [17].

\section{CONCLUSIONS}

The study found that patients with preeclampsia, the expression level of DJ-1 in placental tissues were significantly higher than those of normal pregnant women, and studies have found that DJ-1 is mainly expressed in closely related to the pathogenesis of preeclampsia trophoblast cells, that increased expression of DJ-1 and oxidative stress may be involved in the pathogenesis of preeclampsia. The elevated DJ-1 level may be one of the important factors to preeclampsia. The study also found that preeclampsia umbilical cord blood DJ-1 levels were significantly increased, and there is significant correlation with neonatal body mass, human umbilical cord blood DJ-1 can affect fetal growth and development status. Through the study of DJ-1 in the pathogenesis of preeclampsia, contribute to a more in-depth understanding of eclampsia the mechanism for the prevention and treatment of disease, to develop new ideas.

\section{Funding}

National Health and Family Planning Commission Science Foundation(2019-WJ-04)

Fujian Science and Technology Project(2018Y0005)
Fujian Provincial Health Technology Project(2017-CX-11) Key Clinical Specialty Discipline Construction of Fujian ,P.R.C ([2015] no. 593)

\section{REFERENCES}

1. $\mathrm{Li} \mathrm{H}, \mathrm{Ge} \mathrm{Q}$, Guo Li, et al. Maternal plasma miRNAs expression in preeclamptic pregnancies. Biomed Res Int. 2013; 2013: 970265, doi: 10.1155/2013/970265, indexed in Pubmed: 24195082.

2. Kwiatkowski S, Kwiatkowska E, Rzepka R, et al. Development of a focal segmental glomerulosclerosis after pregnancy complicated by preeclampsia: case report and review of literature. J Matern Fetal Neonatal Med. 2016; 29(10): 1566-1569, doi: 10.3109/14767058.2015.1053865, indexed in Pubmed: 26067260.

3. Anderson UD, Olsson MG, Kristensen $\mathrm{KH}$, et al. Review: Biochemical markers to predict preeclampsia. Placenta. 2012; 33 Suppl: S42-S47, doi: 10.1016/j.placenta.2011.11.021, indexed in Pubmed: 22197626.

4. Zhou X, Zhang Gy, Wang J, et al. A novel bridge between oxidative stress and immunity: the interaction between hydrogen peroxide and human leukocyte antigen $\mathrm{G}$ in placental trophoblasts during preeclampsia. Am J Obstet Gynecol. 2012; 206(5): 447.e7-447.16, doi: 10.1016/j. ajog.2012.03.013, indexed in Pubmed: 22542123.

5. Kwon HS, Hwang HS, Sohn IS, et al. Expression of DJ-1 proteins in placentas from women with severe preeclampsia. Eur J Obstet Gynecol Reprod Biol. 2013; 168(1): 40-44, doi: 10.1016/j.ejogrb.2012.12.024, indexed in Pubmed: 23347603.

6. Xing X. Obstetrics and gynecology [M]. eighth edition. People's Medica Publishing House, Beijing 2013: 64-71.

7. Yan Jy, Jiang LI. Expression of advanced glycation end products in placenta and concentration in maternal and umbilical serum in pre-eclampsia. J Obstet Gynaecol Res. 2015; 41(6): 843-849, doi: 10.1111/jog.12651, indexed in Pubmed: 25656432.

8. Jiang T, Sun Q, Chen S. Oxidative stress: A major pathogenesis and potential therapeutic target of antioxidative agents in Parkinson's disease and Alzheimer's disease. Prog Neurobiol. 2016; 147: 1-19, doi: 10.1016/j. pneurobio.2016.07.005, indexed in Pubmed: 27769868

9. Holmström KM, Baird L, Zhang Y, et al. Nrf2 impacts cellular bioenergetics by controlling substrate availability for mitochondrial respiration. Biol Open. 2013; 2(8): 761-770, doi: 10.1242/bio.20134853, indexed in Pubmed: 23951401.

10. Poston L, Igosheva N, Mistry HD, et al. Role of oxidative stress and antioxidant supplementation in pregnancy disorders. Am J Clin Nutr. 2011; 94(6 Suppl): 1980S-1985S, doi: 10.3945/ajcn.110.001156, indexed in Pubmed: 21613560.

11. Burton GJ, Yung HW, Cindrova-Davies T, et al. Placental endoplasmic reticulum stress and oxidative stress in the pathophysiology of unexplained intrauterine growth restriction and early onset preeclampsia. Placenta. 2009; 30 Suppl A: S43-S48, doi: 10.1016/j.placenta.2008.11.003, indexed in Pubmed: 19081132

12. Ding D, Scott NM, Thompson EE, et al. Increased protein-coding mutations in the mitochondrial genome of African American women with preeclampsia. Reprod Sci. 2012; 19(12): 1343-1351, doi: 10.1177/1933719112450337, indexed in Pubmed: 22902742.

13. Fujimaki $A$, Watanabe $K$, Mori $T$, et al. Placental oxidative DNA damage and its repair in preeclamptic women with fetal growth restriction. Placenta. 2011; 32(5): 367-372, doi: 10.1016/j.placenta.2011.02.004, indexed in Pubmed: 21435716.

14. Zhou X, Zhang Gy, Wang J, et al. A novel bridge between oxidative stress and immunity: the interaction between hydrogen peroxide and human leukocyte antigen $\mathrm{G}$ in placental trophoblasts during preeclampsia. Am J Obstet Gynecol. 2012; 206(5): 447.e7-447.16, doi: 10.1016/j. ajog.2012.03.013, indexed in Pubmed: 22542123.

15. Clements CM, McNally RS, Conti BJ, et al. DJ-1, a cancer- and Parkinson's disease-associated protein, stabilizes the antioxidant transcriptional master regulator Nrf2. Proc Natl Acad Sci U S A. 2006; 103(41): 15091-15096, doi: 10.1073/pnas.0607260103, indexed in Pubmed: 17015834.

16. Salomone F, Li Volti G, Vitaglione P, et al. Coffee enhances the expression of chaperones and antioxidant proteins in rats with nonalcoholic fatty liver disease. Transl Res. 2014; 163(6): 593-602, doi: 10.1016/j. trsl.2013.12.001, indexed in Pubmed: 24365744.

17. Gathiram P, Moodley J. Pre-eclampsia: its pathogenesis and pathophysiolgy. Cardiovasc J Afr. 2016; 27(2): 71-78, doi: 10.5830/CVJA-2016-009, indexed in Pubmed: 27213853 